\title{
BILATERAL SECONDARY GLAUCOMA AND SYSTEMIC HYPERTENSION IN MARFAN'S SYNDROME*
}

\author{
BY \\ V. A. F. MARTIN AND ERIC C. COWAN \\ The Benn Hospital, Belfast
}

SincE Marfan (1896) first described the condition of "dolichostenomalie", some 200 cases have been reported in the literature, each with its variation of the original and with some added claim to the unique. That the syndrome is associated with other congenital abnormalities is also widely reported; its occurrence with congenital heart disease was noted by Lloyd (1937) in his review of a number of cases of arachnodactyly, and Loughridge (1958) described several cases in which it was associated with renal lesions.

The following case shows the combination of several rare features in one child, precipitating one ocular castastrophe which was closely followed by the makings of another.

Reversing the usual procedure, we will give a summary here and follow it with the detailed notes and comments.

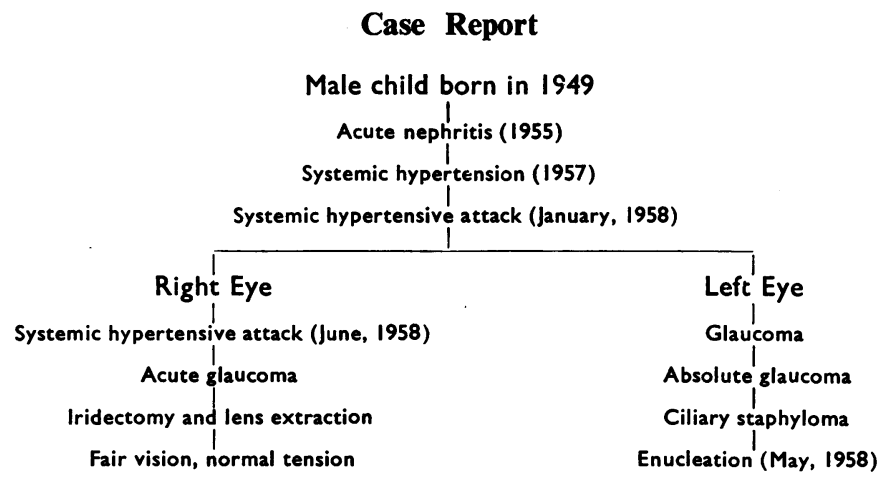

In 1955 a boy, then aged 6 years was admitted to the Royal Belfast Hospital for Sick Children suffering from acute nephritis, and this was treated accordingly.

In December 1956, he was again admitted with a history of vomiting, nausea, pale turns, and headaches; the blood pressure was raised and he was found to have bilateral dislocation of the lens. This seems to have been the first time that any ocular disorder was noted, and the parents did not mention any such suspicion to the admitting doctor. After this the boy was seen to have a rather stamping type of gait, which was put down to his poor eyesight. He was to have been seen by an ophthalmologist at this time but, as the hypertension had settled, he was discharged before this could be done. 
Extensive investigations of the cause of the hypertension were carried out and the only abnormality was that he had a left pelvic kidney. This systemic problem being the main item, the relatively minor ophthalmic lesions were noted but not followed up. About this time, he had his first ophthalmic examination and bilateral dislocation of both lenses was confirmed; no gross disorder was seen in either media or fundi, but details were very hard to make out. A retinoscopy through the lenses showed an error of -12D sph. Glasses were ordered, but not obtained and their effect on vision was therefore never ascertained.

Having contracted Sonne dysentery on December 20 he was transferred to the isolation hospital; his blood pressure being 125/90, anti-hypertensive drugs were continued.

On January 11, 1959, the bowel infection being cleared, he was re-admitted to the Children's Hospital with a blood pressure of 225/180. As the left eye was red one of us was asked to see him.

\section{Ophthalmological Examination}

Left Eye.-There was corneal oedema, ciliary injection, and a widely dilated fixed pupil. There was no perception of light and the picture was that of absolute glaucoma. The lens was behind the iris. After being treated with eserine drops and Diamox tablets (tabs. acetazoleamide) the eye was soft a week later. A ciliary staphyloma was developing and the lens was dislocated downwards and backwards. All this time the right eye remained absolutely quiet with the lens subluxated behind the pupil. The ocular tension was always quite normal.

In March the ciliary staphyloma was more pronounced and, as the tension was again raised, the advisability of enucleation was considered. It was decided to do this when the systemic condition permitted the adminstration of a general anaesthetic, and the operation was performed in mid May.

The pathological report confirmed the diagnosis of intercalary staphyloma and secondary glaucoma. By the beginning of June the socket was quite healed.

Right Eye.-The right lens was found to be dislocated into the anterior chamber, but when the child lay in a dark room, the pupil dilated and the lens returned to its previous position and stayed there.

On June 12, while he was at a convalescent home, the blood pressure again rose sharply to $180 / 190$ systolic and $145 / 150$ diastolic. This was followed by headache, sore eye, nausea, and vomiting. The anti-hypertensive treatment was stepped up and he was transferred next day to the main hospital where he was seen by us the same afternoon. The lens was found to be lying in the pupillary area completely blocking the pupil. The ocular tension was very high and the cornea oedematous, and the lens was gripped firmly by the pupil, lying half in the anterior chamber and half behind the pupil in the posterior chamber. A drop of 1 per cent. eserine was instilled to attempt to close the pupil behind the lens, but this was without effect because of the mechanical position of the lens itself. It was then decided to dilate the pupil to see if that would free the lens, and homatropine and adrenalin (each used in 1 per cent. solution) were used half-hourly combined with "Diamox" orally. The child's general condition was very poor, the blood pressure being still high, and he was heavily sedated with anti-hypertensive drugs.

This treatment was continued throughout the afternoon, and at $6.30 \mathrm{p} . \mathrm{m}$. as the eye was unchanged, it was reluctantly decided to operate and remove the offending lens. This was done under general anaesthetic, the incision being made with a Bard-Parker knife under a large conjunctival flap and extended with scissors. Immediately the anterior chamber was entered some vitreous and iris prolapsed. After these had been excised the lens was removed with the vectis and the wound was closed with a continuous conjunctival suture without further loss of vitreous. 
2 weeks later, the eye having healed well, the patient was sent back to the convalescent home. The blood pressure was now 155/105. The ocular tension was normal, but owing to medial haze no fundus reflex was obtained on attempted retinoscopy. The visual acuity was $6 / 36$ with a $+10 \mathrm{D}$ sph.

A month later the right eye was quite quiet, the ocular tension was normal, the media were clear enough to see the disc, and a refraction was obtained. As the child could not keep his eye still, it was difficult to make out fundus details clearly, but even so no haemorrhages or exudates were present, the disc was normal, and some central retinal degenerative changes were seen.

With $+12 \mathrm{D}$ sph. he could recognize different nurses about the room and wearing these glasses 2 months later could read 6/24-6/18.

\section{Comment}

In this case of Marfan's syndrome there appeared to be a connexion between the elevation of the systemic blood pressure to a high level, and the intra-ocular glaucomatous crisis. The fact that there was also a congenitallydisplaced kidney is noted. That the intra-ocular pressure varies with the hydrostatic pressure in the ocular capillaries is well known, but it does not usually vary with the general arterial blood pressure, systemic hypertension not being essential for glaucoma and the occurrence of the two together being accidental rather than essential. But it is also known that, in the hypertensive individual, the high blood pressure may possibly break through the peripheral resistance of the arterioles so that a hypertensive crisis may well give rise to an acute rise in the ocular tension. The raised systemic pressure may also make the intra-ocular neuro-vascular controlling mechanism more labile and more subject to trauma from a subluxated lens as in this case. That the two ocular incidents recorded above were preceded and accompanied by systemic hypertensive crisis could suggest a connexion between them, in as much as the increased blood pressure could have started off a chain of incidents which in the first case led to the closure of the filtration angle with peripheral anterior synechiae and in the second to the blockage of the pupil by the dislocated lens.

Hilding (1957) stated that subluxation of one or both lenses in a child presents the ophthalmologist with a problem entailing a difficult decision, and Agnew (1885) admitted that such cases are embarrassing. . We would go further and say that; having seen the results that may ensue, and the rapidity with which they may happen, such cases are not only difficult and embarrassing but are some of the most worrying of all ophthalmic emergencies. Hilding (1957) described two cases of glaucoma in a child due to blockage of the pupils by subluxated lenses in which he was able to delay operation for some days, but in this case we felt delay to be out of the question, as the first eye had gone blind in a couple of days.

Apart from the possible systemic cause in this case, few cases are recorded in the literature in which blockage of the pupil by the gripping of the lens by the pupillary sphincter gave rise to glaucoma. The cases reported by Chandler (1951) and Hilding (1957) are the main examples. 
Duke-Elder (1940) stated that a completely dislocated lens could cause glaucoma by giving rise to a derangement and stasis of the circulation excited by neuro-vascular reflexes set up by the mechanical irritation of the iris and ciliary body.

The choice of medical treatment in cases of this type is helped by our experience. Diamox has not proved to be of great use in cases of secondary glaucoma with peripheral anterior synechiae, only slight or transient relief being described in the report by Grant and Trotter (1954). Like Hilding we tried a miotic without result, and, the subsequent use of mydriatics having achieved nothing, we had to resort to surgery.

Duke-Elder (1940) suggested that miotics should be avoided, that they might precipitate an attack of glaucoma, and that a mydriatic could bring about a marked improvement, i.e. glaucoma inversum. A change in position and massage of the eye, as suggested by Bailliart (1922) and Saito (1934), might also help but in this case even the use of mydriatics failed to release the lens from the grasp of the sphincter. In the absence of a prompt response to medical treatment, it is obvious that the only effective treatment is to remove the lens as soon as possible, and Diamox may be useful as a preoperative measure. We had to give Diamox orally by tube as no parenteral preparation was available to us at that time. As the child was semi-conscious and often sick, very little of it must have been absorbed.

Hilding (1957) was able to remove the lens in one of his cases with intracapsular forceps, but had to use the vectis in the other case. With the initial vitreous loss and the position of the lens we used the loop without attempting to grip the lens with forceps. Spaeth (1948) suggested that the pupil should be fully contracted behind the lens before operation to prevent the lens falling backwards. This again was impossible here because of the position of the lens. Spaeth also advised against iridectomy, but with the initial iris prolapse this had to be done; Philps (1950) was in full agreement with wide iridectomies in such cases.

\section{Conclusion}

Secondary glaucoma was caused by a dislocated lens in the pupillary area in a child who had lost one eye after a similar previous upset. He was in the middle of a systemic hypertensive attack and after a short trial of medical therapy surgical operation was resorted to. The eye responded well and useful sight was preserved. Hilding (1957) wrote: "If confronted with another similar situation, the same dilemma would obtain ... should the lens be removed or not". Seeing the favourable outcome in a case in which so many operative complications could have arisen but did not, we offer our case for the perusal of those who may be confronted with a similar one in the future.

\footnotetext{
We should like to thank Prof. F. M. B. Allen, Nuffield Professor of Child Health in the Queen's University of Belfast, for permitting access to the patient's medical records.
} 


\section{REFERENCES}

Agnew, C. R. (1885). Trans. Amer. ophthal. Soc., 4, 69.

Bailliart, P. (1922). Bull. Soc. Ophtal. Paris, 35, 98.

Chandler, P. A. (1951). A.M.A. Arch. Ophthal, 45, 125.

DUKE-ElDER, S. (1940). "Text-book of Ophthalmology", vol. 3, p. $3309 . \quad$ Kimpton, London.

Grant, W. M., and Trotter, R. R. (1954). A.M.A. Arch. Ophthal., 51, 735.

HildING, A. C. (1957). Ibid., 57, 33.

LLOYD, R. I. (1937). Arch. Ophthal. (Chicago), 17, 66.

LOUGHRIDGe, L. (1958). Personal communication.

Marfan, B.-J. A. (1896). Bull. Soc. méd. Hôp. Paris., 3 sér., 13, 22.

PHILPS, S. (1950). "Ophthalmic Operations", p. 249, Baillière, Tindall and Cox, London.

SAITo, S. (1934). Chuo-Ganka-Iho, 26, No. 4. (Ref. Zbl. ges. Ophthal., 31, 642).

SpaETH, E. B. (1948). "Principles and Practice of Ophthalmic Surgery", 4th ed., p. 701. Lea and Febiger, Philadelphia. 